YIIK 5I7. 5
Н.И.Волкова, М.В.Ковтун

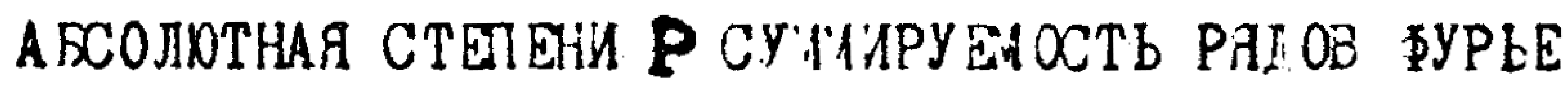
YETON OI XAYCAOP DA

Последоват 'яьность $\{\operatorname{tn}(A(x))\}$, где

$$
\left.\operatorname{tn}(A(x))=\sum_{k=0}^{n}\left(\begin{array}{l}
n \\
k
\end{array}\right)\right\lrcorner^{n-k} \mu_{k} S_{k}(x), S_{k}(x)=\sum_{v=0}^{k} A_{v}(x),
$$

называется [I] преобразованием Хаусдорфа ряда $\sum_{k=0}^{\infty} A_{k}(x)$, где $\left\{\mu_{n}\right\}$ эаданная последовательность вещественных или комплексних чиоел, в $\Delta^{v} \mu_{k}$ озна ает разность неотрицательного целого. орядка $\nu$ последоэательности $\left\{\mu_{k}\right\}$, т.е.

$$
\Delta^{0} \mu_{k}=\mu_{k}, \Delta^{v} \mu_{k}=د^{v-1} \mu_{k}-\Delta^{v-1} \mu_{k+1}, v \geqslant 1 .
$$

Ряд $\sum_{k=0}^{\infty} A_{k}(x)$ сумиируем ме.лдом Хаусдорда к рункции $S(x)$ или $\left(H, \mu_{n}\right)$-суммируем н. $S(x)$, зсли оуществует $\lim _{n \rightarrow \infty} t_{n}(f(x))=S(x)$.

Говорят [2], что ряд $\sum_{k=0}^{\infty} A_{k}(x)$ вбсолотно степенк $p+1$ суммируем методом Хауслорфа или $\left|H, \mu_{n}\right|_{p}$-суммируеч, если

$$
\left.\sum_{=1}^{\infty} n^{p-1} \mid \operatorname{tn}\left(A^{\prime}: \therefore\right)\right)-\left.\operatorname{thn}_{n-1}(A(x))\right|^{p}-\infty \text {. }
$$

Заметим, что метод Чезаро $(C, d)$ вляется метод: I Хаусдорра $\left(H, 1 / \mathrm{Ah}^{\alpha}\right)$ при лобом $\alpha \neq-1,-2, \ldots$. метод Гельдера $H^{\alpha}$ явдяет яя методом Хаусдпра $\left(H, 1 /(n+1)^{\alpha}\right)$, метод Эйера $(E, q)$ является методом Хаусдорра $\left(H, 1 /(1+q)^{n}\right)$, обобщённий зтод чезаро $(C, \alpha, \beta)$ является методом Хаусдорд $\left(H, A_{n}^{\beta} / A_{n}^{\alpha+\beta}\right)$ при побнх $\alpha \neq-1,-2, \ldots n \alpha+\beta \neq-1,-2, \ldots$.

для того чтобн $\left(H, \mu_{n}\right)$-метод бнл хонсервативнн, необходимо и достаточно, чмобн существовала рункция $X(x)$ ограниченно варивции Ha $(0,1)$ таквя, что

$$
\mu_{n}=\int_{0}^{1} x^{n} d X(x), n=0,1,2, \ldots
$$

Hе нарушая обғности доказательстяв, бу зм считать, что $X(0)=0$. Если $X(+0)=X\left(0_{1}=0, X(1)=1\right.$, го $\left(H, \mu_{n}\right)$-метол являетоя регуI пным.

Известн [3], что если $\left(H, \mu_{n}\right)$ являетея консервативним или

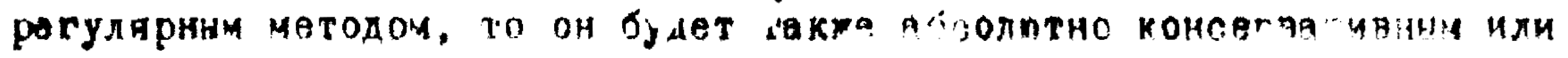
accosoth perymaphas meтодом. 
$-87-$

Пусть $f(t)-2 \pi$-периодическая функция, интегрируемая в смисле Лебега на $(-\pi, T)$. Положим, что её ряд ђурье ияеет вид

$$
\sum_{n=1}^{\infty}\left(a_{n} \cos n t+b_{n} \sin n t\right) \equiv \sum_{n=1}^{\infty} A_{n}(t) \text {. }
$$

Сопряжённим рядом Бу. ье является няд

$$
\sum_{n=1}^{\infty}\left(a_{n} \sin n t-b_{n} \cos n t\right) \equiv-\sum_{n=1}^{\infty} B_{n}(t)
$$

Продитпреренцированным рядом к ряду (I) является ряд

$$
\sum_{n=1}^{\infty} n\left(b_{n} \cos n t-a_{n} \sin n t^{\prime} \equiv \sum_{n=1}^{\infty} n B_{n}(t)\right. \text {. }
$$

- Продитуеренцированным рядом к ряду(2) является ряд

$$
\sum_{n=1}^{\infty} n\left(a_{n} \cos n t+b_{n} \sin n t\right) \equiv \sum_{n=1}^{\infty} n A_{n}(t) \text {. }
$$

ізедё'я следурщие обозначения:

$$
\begin{array}{cr}
\varphi(t)=\frac{1}{2}[f(x+1)+f(x-t)], & \psi(t)=\frac{1}{2}[f(x+t)-f(x-t)] ; \\
\varphi_{1}(t)=\frac{1}{t} \int_{0}^{t}(n(u) d u, & \Psi_{1}(t)=\frac{1}{t} \int_{0}^{t} \psi(u) d u .
\end{array}
$$

Ооознвчим ряд (I) через $F(t)$, ряд (2) через $\widetilde{F}(t)$, рям (3) 4ерез. $\mathcal{F}^{\prime}(t)$ и ряд (4) через $\widetilde{F}^{\prime}(t)$.

Имерт место следующие теоремн.

Теоремв I. Если $\left.\int_{0}^{\pi}\left|\left(\frac{\varphi_{1}(t)}{t}\right)^{\prime}\right|\right|^{P} d t<\infty$, а метоп $\left(H, \mu_{n}\right)$ янляетоя консервативним и $\int_{0}^{1} \frac{|d| x(x) \mid}{1-x}<\infty$, то ояд вурье бункцин $f(t)$ при $t=x$ $\left|H, \mu_{n}\right|_{0}$-cymupyeir.

Iоказательство. Дая ряда Бурье функиии $f(x)$

$$
\begin{gathered}
\operatorname{Sn}_{n}(x)=\frac{2}{\pi} \int_{0}^{\pi} \varphi(t) \cos n t d t= \\
=\frac{2}{\pi} \int_{0}^{\pi}\left(t^{2} \cos n t-\frac{2 t}{n} \sin n t-\frac{2}{n^{2}}(\cos n t-1)\right) d\left(\frac{\varphi_{1}(t)}{t}\right)+\frac{4}{\pi^{2}} \varphi_{0}(\pi) \frac{\cos n \pi}{n^{2}} .
\end{gathered}
$$

llокажем, что рал (I) $\left|\mathrm{H}, \mathrm{j}_{\mathrm{n}}\right|_{\mathrm{p}}$-сумми ууем, т. е.

$$
\sum_{n=1}^{\infty} n^{p-1}\left|t_{n}(F(x))-t_{n-1}(F(x))\right|^{p}<\infty .
$$

$\ln x$ kak

$$
\operatorname{tn}(F(x))-\operatorname{tn-1}(F(x))=\sum_{k=1}^{n}\left(\begin{array}{l}
n-1 \\
k-1
\end{array}\right) \Delta^{n-k} \mu_{k} I_{k}(x)=
$$


$-88-$

$$
=\int_{0}^{\pi} g(n, t) d\left(\frac{\varphi_{1}(t)}{t}\right)+\frac{4}{\pi^{2}} \varphi_{1}(\pi) \sum_{k=1}^{n}\left(\begin{array}{l}
n-1 \\
k-1
\end{array}\right) \Delta^{n-k} \mu_{k} \frac{\cos k \pi}{k^{2}},
$$

rä

$$
g(n, t)=\frac{2}{\pi} \sum_{k=1}^{n}\left(\begin{array}{l}
n-1 \\
k-1
\end{array}\right) \Delta^{n-k} \mu_{k}\left(t^{2} \cos k t-\frac{2 t}{k} \sin k t-\frac{2}{k^{2}}(\cos k t-1)\right),
$$

то силу неравенотва Минковского получим

$$
\begin{aligned}
& \sum_{n=1}^{N} h^{p-1}\left|t_{n}(F(x))-t_{n-1}(F(x))\right|^{p} \leq\left\{\left(\sum_{n=1}^{N} n^{p-1}\left|\int_{\theta}^{\pi} g(n, t) d\left(\frac{\varphi_{1}(t)}{t}\right)\right|^{p}\right)^{1 / p}+\right. \\
& \left.+\left(\sum_{n=1}^{N} h^{p-1}\left|\frac{4}{\pi^{2}} \varphi_{1}(\pi) \sum_{k=1}^{n}\left(\begin{array}{c}
n-1 \\
k-1
\end{array}\right) \Delta^{n-k} \mu_{k} \frac{\cos k \pi}{k^{2}}\right|^{p}\right)^{\frac{1}{p}}\right\}^{p} .
\end{aligned}
$$

Далее, применяя норавенотво Гехьдера дия р>1,

$$
\begin{aligned}
& \sum_{k=1}^{N} n^{p-1}\left|\frac{4}{\pi^{2}} \varphi_{1}(\pi) \sum_{k=1}^{n}\left(\begin{array}{l}
n-1 \\
k-1
\end{array}\right) \Delta^{n-k} \mu_{k} \frac{\cos k \pi}{k^{2}}\right|^{p} \leq \\
& \leq \mathcal{K} \sum_{n=1}^{N} \sum_{k=1}^{n}\left(\begin{array}{l}
n-1 \\
k-1
\end{array}\right) \mid \Delta^{n-k} j \cdot 1 \frac{1}{k^{2}}\left(n \sum_{k=1}^{n}\left(\begin{array}{c}
n-1 \\
k-1
\end{array}\right)\left|\Delta^{n-k} \mu_{k}\right| \frac{1}{k^{2}}\right)^{R-1} \leq \\
& \left.\leq \mathcal{K} \sum_{k=1}^{\infty} \frac{1}{k^{2}} \sum_{n=k}^{\infty}\left(\begin{array}{l}
n \\
k
\end{array}\right) \mid \Delta^{n-k} \mu_{k}\right)\left(\sum_{k=1}^{n}\left(\begin{array}{l}
n \\
k
\end{array}\right)\left|\Delta^{n-k} \mu_{k}\right|\right)^{p-1}<\infty
\end{aligned}
$$

$$
\left.\sum_{n=1}^{N} n^{R}\left|\int_{0}^{\pi} g(n, t) d\left(\frac{\varphi_{1}(t)}{t}\right)\right|^{p} \leq \int_{0}^{\pi}\left|\left(\frac{\varphi_{1}(t)}{t}\right)\right|^{p} d t \sum_{n=1}^{N}|g(n, t)|\left|\int_{0}^{R} n\right| g(n, t) \mid d t\right)^{R-1} .
$$

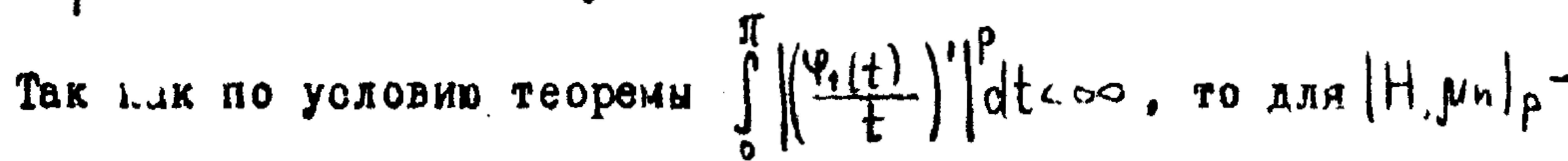
оуммируемости ряда Фурье достаточно показать, что

I) $\sum_{n=1}^{\infty}|g(n, t)| \leq \mathcal{K}$;

2) $\ln |g(1, t)| d t \leq \pi$,

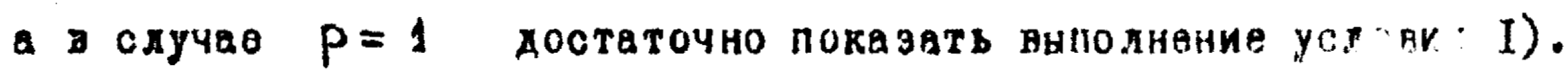
Pacenot pим

$$
\sum_{n=1}^{\infty}|g(n, t)| \leqslant \frac{2}{\pi} \sum_{n=1}^{\infty}\left|\sum_{k=1}^{n}\left(\begin{array}{l}
n-11 \\
k-1
\end{array}\right) \Delta^{n-k} \mu_{k} t^{2} \cos k t\right| .
$$


$-89-$

$$
\begin{gathered}
+\frac{4}{\pi} \sum_{n=1}^{\infty}\left|\sum_{k=1}^{n}\left(\begin{array}{l}
n-1 \\
k-1
\end{array}\right) \Delta^{n-k} \mu_{k} t \frac{\sin k t}{k}\right|+\frac{4}{\pi} \sum_{n=1}^{\infty}\left|\sum_{k=1}^{n}\left(\begin{array}{c}
n-1 \\
k-1
\end{array}\right) \Delta^{n-k} \mu_{k} \frac{\cos k t-1}{k-1}\right|= \\
=\sum_{1}+\sum_{2}+\sum_{3} . \\
\operatorname{Tax} \operatorname{xax} \mu_{n}=\int_{0}^{1} x^{n} d x(x), n=0,1,2, \text {. To } \Delta^{n-k} \mu_{k}=\int_{0}^{1}(1-x)^{n-k} x^{k} d x(x) \\
\sum_{k=1}^{n}\left(\begin{array}{l}
n-1 \\
k-1
\end{array}\right) \Delta^{n-k} \mu_{k} \cos k t=\operatorname{Re} \int_{0}^{1} x e^{i t}\left(1-x+x e^{i t}\right)^{n-1} d x(x)= \\
=\int_{0}^{1} x \rho^{n-1} \cos (t+(n-1) \varphi) d x(x),
\end{gathered}
$$

Ine $p^{2}=1-4 x(1-x) \sin ^{2} \frac{t}{2} ; \varphi=\operatorname{arctg} \frac{x \sin t}{1-x+x \cos t}$, to

$$
\begin{aligned}
& \sum_{1} \leq X t^{2} \sum_{n=1}^{\infty} \int_{0}^{1} x \rho^{n-1}|d x(x)|=X t^{2} \int_{0}^{1} x|d x(x)| \sum_{n=1}^{\infty} \rho^{n-1}= \\
& =K t^{2} \int_{0}^{1} x|d x(x)| \frac{1+\rho}{1-\rho^{2}} \leqslant K t^{2} \int_{0}^{1} \frac{x|d x(x)|}{x(1-x) \sin ^{2} t_{2}} \leqslant K t^{2} \int_{0}^{1} \frac{|d x(x)|}{(1-x) t^{2}}<\infty \text {; } \\
& \sum_{2}=\frac{4}{\pi} t \sum_{n=1}^{\infty}\left|\sum_{k=1}^{n}\left(\begin{array}{c}
n-1 \\
k-1
\end{array}\right) \Delta^{n-1} \mu_{k} \int_{0}^{t} \cos x u d u\right|=\frac{4}{\pi} t \sum_{n=1}^{\infty}\left|\int_{t}^{\pi} \sum_{k=1}^{n}\left(\begin{array}{c}
n-1 \\
k-1
\end{array}\right) \Delta^{n-k} \mu_{k} \cos k u d u\right| \leqslant \\
& \leq \frac{4}{\pi} t \int_{t}^{\pi} \sum_{n=1}^{\infty}\left|\sum_{k=1}^{n}\left(\begin{array}{l}
n-1 \\
k-1
\end{array}\right) \Delta^{n-k} \mu_{k} \cos x u\right| d u \leq \pi t \int_{t}^{\pi} d u \int_{0}^{1} \frac{|d x(x)|}{(1-x) u-<\infty, 0<t<\pi ;} \\
& \sum_{3}=\frac{4}{\pi} \sum_{n=1}^{\infty} \sum_{k=1}^{n}\left(\begin{array}{c}
n-1 \\
k-1
\end{array}\right)\left|\Delta^{n-k} \mu_{k}\right| \frac{2}{k^{2}} \leq \mathcal{K} \sum_{k=1}^{\infty} \frac{1}{k^{2}} \sum_{n=k}^{\infty}\left(\begin{array}{c}
n-1 \\
k-1
\end{array}\right)\left|\Delta^{n-k} \mu_{k}\right| \leq \mathcal{K} .
\end{aligned}
$$

Итак, усховия I) виполнено.

Расамотрим

$$
\begin{aligned}
& \int_{0}^{\pi} n|g(n, t)| d t \leq \frac{2 n}{\pi} \int_{0}^{\pi}\left|\sum_{k=1}^{n}\left(\begin{array}{c}
n-1 \\
k-1
\end{array}\right) \Delta_{0}^{n-k} \mu_{k} t^{2} \cos k t\right| d t+\frac{4 n}{\pi} \int_{0}^{\pi}\left|\sum_{k=1}^{n}\left(\begin{array}{c}
n-1 \\
k-1
\end{array}\right) \Delta^{n-k} \mu_{k} \frac{t \sin k t}{k}\right| d t+ \\
& \left.+\frac{4 n}{\pi} \int_{0}^{\pi} \mid \sum_{k=1}^{n} \begin{array}{c}
n-1 \\
i-1
\end{array}\right) \Delta^{n-k} \mu_{k} \frac{\cos k_{i}-1}{k^{2}}\left|d t \leq \pi n \int_{0}^{\pi} t^{2} d t \int_{0}^{1} x \rho^{n-1}\right| d x(x) \mid+
\end{aligned}
$$


$-90-$

$$
\begin{aligned}
& +\mathcal{K} n \int_{0}^{\pi} t d t \int_{t}^{\pi}\left|\sum_{k=1}^{n}\left(\begin{array}{l}
n-1 \\
k-1
\end{array}\right) \Delta^{n-k} \mu_{k} \cos k u\right| d u+K \int_{0}^{\pi} d t \sum_{k=1}^{n}\left(\begin{array}{l}
n \\
k
\end{array}\right)\left|\Delta^{n-k} \mu_{k}\right| \leq \\
& \leq K \int_{0}^{1} \frac{|d x(x)|}{1-x} \int_{0}^{\pi} t^{2} n \rho^{n-1} d t+\int_{0}^{1} \frac{|d x(x)|}{1-x} \int_{0}^{\pi} n \rho^{n-1} d u \int_{0}^{u} t d t+K \leq K
\end{aligned}
$$

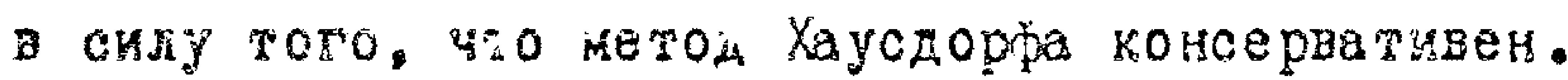

Тек как усяовия I) и 2) виполнябтея, то ряд (I) $|H, \mu n|_{p}-$ сумหnpyen.

Creдствие I [4] . Eсли $\int_{0}^{\pi}\left(\mid \frac{\varphi t t}{t}\right)^{\prime} \mid d t<\infty$, то pяд (I) $|E, q|_{p}^{p}$ суния туен при $t=x$.

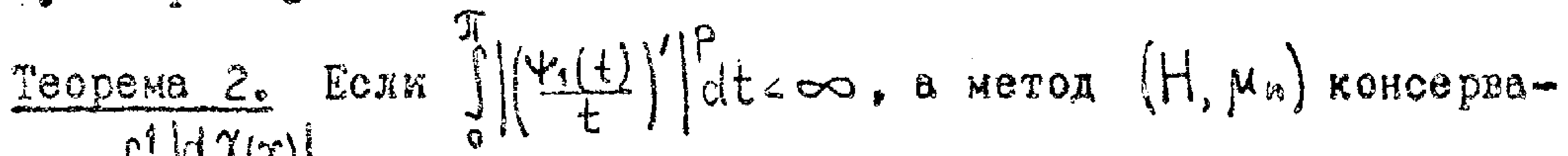
тивен : $\int_{0}^{1} \frac{|d \gamma| x||}{1-x}<\infty$, то сопряжённни ряд фурье функции $f(t)$ при $t=x \quad\left|H_{1}, \mu_{n}\right|_{p}-$-уквируем.

Докезательетво. ДАл сопряжённого ряда фурье функции $f(x)$

$$
B_{n}(x)=\frac{2}{\pi} \int_{0}^{\pi} \psi(t) \sin n t d t=
$$

$=\frac{2}{\pi} \int_{0}^{\pi}\left(t^{2} \sin n t+\frac{2 t}{n} \cos n t-\frac{2}{n^{2}} \sin n t\right) d\left(\frac{\psi_{1}\left(t^{\prime}\right)}{t}\right)-\frac{4}{\pi n} \psi_{1}(\pi) \cos n \pi$.

Поважем, чта ряд (2) $\left|H_{3} \mu_{r}\right|_{p}$-суммкруем.

Tas

$$
\operatorname{tn}(\tilde{F}(x))-\operatorname{tn}_{n-1}(\tilde{F}(x))=\int_{0}^{\pi} \hat{v}\left(n_{0} t\right) d\left(\frac{\psi_{1}(t)}{t}\right)+\frac{4}{\pi} \sum_{k=1}^{n}\left(\begin{array}{c}
n-1 \\
k-1
\end{array}\right) \Delta \mu^{n-k} \mu_{k} \frac{\psi_{1}(\pi)}{k} \cos k \pi,
$$

nae

$$
G(n, t)=\frac{2}{\pi} \sum_{k=1}^{n}\left(\begin{array}{l}
n-1 \\
k-1
\end{array}\right) \Delta^{n-k} \mu_{k}\left(t^{2} \sin k t+\frac{2 t}{k} \cos k t-\frac{2}{k^{2}} \sin k t\right),
$$

то з силу неравенстве Мниковского

$$
\sum_{n=1}^{N} n^{P-1}\left|t_{n}(\widetilde{F}(x))-t_{n-1}(\widetilde{F}(x))\right|^{P} \leqslant\left\{\left(\sum_{n=1}^{N} n^{P-1}\left|\int_{0}^{\pi} G(n, t) d\left(\frac{\psi_{1}(t)}{t}\right)\right|^{P}\right)^{1 / P}+\right.
$$


$-3 I-$

$$
\left.+\left(\sum_{n=1}^{N} n^{p-1}\left|\frac{4}{\pi} \sum_{k=1}^{n}\left(\begin{array}{c}
n-1 \\
k-1
\end{array}\right) \Delta^{n-k} \mu_{k} \frac{\Psi_{1}(\pi)}{k} \cos k \pi\right|^{p}\right)^{1 / p}\right\}^{p}
$$

Прнменяя неравенство Гельдера для $p>1$, имеем

$$
\begin{aligned}
& \sum_{n=1}^{N} \cdot P^{p-1}\left|\frac{4}{\pi} \sum_{k=1}^{n}\left(\begin{array}{c}
n-1 \\
k-1
\end{array}\right) \Delta^{n-k} \mu_{k} \frac{\psi_{1}(\pi)}{k} \cos k \pi\right|^{p}= \\
\leq & \sum_{k=1}^{N}\left|\sum_{k=1}^{n}\left(\begin{array}{c}
1-1 \\
k-1
\end{array}\right) \Delta^{n-k} \mu_{k} \frac{\cos k \pi}{k}\right|\left|\sum_{k=1}^{n} n\left(\begin{array}{c}
n-1 \\
k-1
\end{array}\right) \Delta^{n-k} \mu_{k} \frac{\cos k \pi}{k}\right|^{p-1} \leq K
\end{aligned}
$$

B

$$
\sum_{n=1}^{N} n^{P-1}\left|\int_{0}^{\pi} G(n, t) d\left(\frac{\Psi_{1}(t)}{t}\right)\right|^{P} \leq\left.\int_{0}^{\pi}\left|\left(\frac{\Psi_{1}(t)}{t}\right)^{\prime}\right| d t \sum_{n=1}^{N} G(n, t)|| \int_{0}^{\pi} n|G(n, t)| d t\right|^{R_{1}} .
$$

Так как по условио теоремн $\int_{0}^{\pi}\left|\left(\frac{\psi_{1}(t)}{t}\right)^{\prime}\right|^{p} d t<\infty$, то для $\left|H_{1} \mu_{h}\right|_{p}$ суммируемости ряда (2) достаточно показать, что

3) $\sum_{n=1}^{\infty}|G(n, t)| \leq \mathscr{K}$;

4) $\int_{0}^{\pi} h|G(n, t)| d t \leq \mathcal{X}$

а в случае $P=1$ достатс но показать вмполнение условия 3 ).

Рвссмотрим

$$
\begin{aligned}
& \sum_{n=1}^{\infty}|-(n, t)| \leq \frac{2}{\pi} \sum_{k=1}^{\infty}\left|\sum_{k=1}^{n}\left(\begin{array}{l}
n-1 \\
k-1
\end{array}\right) \Delta^{n-k} \mu_{k} t^{2} \sin k t\right|+\frac{4}{\pi} \sum_{n=1}^{\infty}\left|\sum_{k=1}^{n}\left(\begin{array}{c}
n-1 \\
k-1
\end{array}\right) \Delta^{n-k} \mu_{k} t \frac{\cos k t}{k}\right|+ \\
& +\frac{4}{\pi} \sum_{n=1}^{\infty}\left|\sum_{k=1}^{n}\left(\begin{array}{c}
n-1 \\
k-1
\end{array}\right) \Delta^{n-k} \mu_{k} \frac{n^{\prime} n k t}{k^{2}}\right|=\sum_{1}^{\prime}+\sum_{2}^{\prime}+\sum_{3}^{\prime} .
\end{aligned}
$$

Tak kak

$$
\left|\sum_{k=1}^{n}(k-1) \Delta^{n-k} \mu_{k} \sin k t\right|=\left|\int_{0}^{1} x \rho^{n-1} \sin (t+(n-1) \varphi) d x(x)\right| \leqslant \int_{0}^{1} x \rho^{-1}|d x(x)| \text {, }
$$

то, рассужда как при докауательстве төоремн I. плдучия

$$
\begin{aligned}
& \sum^{\prime} \leq \mathcal{K} t^{2} \sum_{n=1}^{\infty}\left|x \rho^{n-1}\right| d x(x) \mid \leq \mathcal{K} \int_{0}^{1} \frac{|d x(x)|}{1-x} \leq \mathcal{K}, \\
& \Gamma_{-2}^{\prime}=\frac{4 t}{\pi} \sum_{n=1}^{\infty}\left|\sum_{k=1}^{n}\left(\begin{array}{c}
n-1 \\
k-1
\end{array}\right) \Lambda^{n-k} \mu_{k}\left(\int_{t}^{\pi} \sin k u d u+\frac{\cos k \pi}{k}\right)\right| \leq
\end{aligned}
$$


$-92-$

$$
\begin{aligned}
& \leq \frac{4 t}{\pi} \sum_{n=1}^{\infty}\left|\sum_{k=1}^{n}\left(\begin{array}{l}
n-1 \\
k-1
\end{array}\right) \Delta^{n-k} \mu_{k} \int_{t}^{\pi} \sin k u d u\right|+\frac{4 t}{\pi} \sum_{k=1}^{\infty}\left|\sum_{k=1}^{n}\left(\begin{array}{c}
n-1 \\
k-1
\end{array}\right) \Delta^{n-k} \mu_{k} \frac{\cos x \pi}{k}\right| \epsilon \\
& \leq \mathcal{K} t \sum_{n=1}^{\infty} \int_{t}^{\pi} d u \int_{0}^{1} x \rho^{n-1}|d x(x)|+\mathcal{H}^{\prime} \sum_{n=1}^{\infty} \frac{1}{n} \int_{0}^{1}\left|(1-2 x)^{n}-(1-x)^{n}\right||d x(x)| \leq \\
& \leq K t \int_{t}^{\pi} d u \int_{0}^{1} x \sum_{n=1}^{\infty} \rho^{n-1}|d x(x)|+\mathcal{K} \int_{0}^{1 / 2}|d x(x)|+\mathcal{K} \int_{\mathbb{t}_{2}}^{1}|d x(x)| \ln \frac{1}{2 x(1-x)} \leq J^{\prime} ; \\
& \sum_{3}^{\prime} \leq \frac{4}{\pi} \sum_{k=1}^{\infty} \frac{1}{k^{2}} \sum_{n=k}^{\infty}\left(\begin{array}{c}
n-1 \\
k-1
\end{array}\right)\left|\Delta^{n-k} \mu_{k}\right| \leq K .
\end{aligned}
$$

Paccmotpus

$$
\begin{aligned}
& \int_{0}^{\pi} n|f(n, t)| d t \leq \frac{2 n}{\pi} \int_{0}^{\pi}\left|\sum_{k=1}^{n}\left(\begin{array}{l}
n-1 \\
k-1
\end{array}\right) \Delta^{n-k} \mu_{k} t^{2} \sin k t\right| d t+\frac{4 n}{\pi} \int_{0}^{\pi}\left|\sum_{k=1}^{n}\left(\begin{array}{l}
n-1 \\
k-1
\end{array}\right) \Delta^{n-k} \mu_{k} \frac{t \cos k t}{k}\right| d t+ \\
& +\frac{4 n}{\pi} \int_{0}^{\pi}\left|\sum_{k=1}^{n}\left(\begin{array}{l}
n-1 \\
k-1
\end{array}\right) \Delta^{n-k} \mu_{k} \frac{\sin k t}{k^{2}}\right| d t \leq \mathcal{K} \int_{0}^{\pi} t^{2} d t \int_{0}^{1} x \rho^{n-1}|d x(x)|+. \\
& \left.+K_{n} \int_{0}^{\pi} t d t\left|\sum_{k=1}^{n}\left(\begin{array}{l}
n-1 \\
k-1
\end{array}\right) \Delta^{n-k} \mu_{k}\right| \int_{t}^{\pi} \sin k u d u+\frac{\cos k \pi}{k}\right)\left|+\mathcal{K} \int_{0}^{\pi} \sum_{k=1}^{n}\left(\begin{array}{l}
n \\
k
\end{array}\right)\right| \Delta^{n-k} \mu_{k} \mid \leq \\
& \leq J_{1} \int_{0}^{1} \frac{|d x(x)|}{1-x} \int_{0}^{\pi} t^{2} n \rho^{n-1} d t+\mathcal{K} \int_{0}^{\pi} t d t \sum_{k=1}^{n}\left(\begin{array}{l}
n \\
k
\end{array}\right)\left|\Delta^{n-k} \mu_{k}\right|+\mathcal{K} \leq \mathcal{K} .
\end{aligned}
$$

Итак, усяовия 3) и 4) внподняотоя, поотому ряд (2) $\left|H, \mu_{n}\right|_{p}-$ оумамируек.

Следствке $2[4]$. Еоли $\int_{0}^{\pi}\left|\left(\frac{\Psi(t)}{t}\right)^{\prime}\right|^{p} d t<\infty$. то ряд (2) $|E, q|_{p}$ суммируем при $t=x$

теорем 3. Если $\int_{0}^{\pi}\left|\left(\frac{\psi(t)}{t^{2}}\right)^{\prime}\right|^{p} d t<\infty$, метод $\left(H, \mu_{n}\right)$ хонсервативен $\int_{0}^{1} \frac{|d x(x)|}{1-x}<\infty$. то pra (3) $\left|H, \mu_{n}\right|_{p}$-оумиируек при $t=x$. 耳ока 3 тельство. Дхя продифферениированного ряда фурье фунхиии $f(x)$ 
$-93-$

$n B_{n}(x)=\frac{2 n}{\pi} \int_{0}^{\pi} \psi(t) \sin n t a_{2}^{\prime}=\frac{2}{\pi} \int_{0}^{\pi}\left(t^{2} \cos n t-\frac{2 t}{n}(\cos n t-1)\right) d\left(\frac{\psi(t)}{t^{2}}\right)$.

$\operatorname{Tax} \operatorname{xax}$

$$
t_{n}\left(F^{\prime}(x)\right)-t_{n-1}\left(F^{\prime}(x)\right)=\int_{0}^{\pi} g(n, t) d\left(\frac{\psi(t)}{t^{2}}\right),
$$

то $>$ оиху неравенства Гельдера для $\beta>1$

$$
\begin{gathered}
\sum_{n=1}^{N} h^{p-1}\left|t_{n}\left(F^{\prime}(x)\right)-t_{n-1}\left(f^{\prime \prime}(x)\right)\right|^{p} \leq \\
\leq \int_{0}^{\pi}\left|\left(\frac{\psi(t)}{t^{2}}\right)^{\prime}\right|^{p} d t \sum_{n=1}^{N}|g(n, t)|\left(\int_{0}^{\pi} h|g(n, t)| d t\right)^{p-1} .
\end{gathered}
$$

Tак как по уеловив теоремв $\left.\int_{0}^{\pi}\left|\left(\frac{\psi(t)}{t^{2}}\right)^{\prime}\right|\right|^{P} d t<\infty$, то, испо :зуя оценки, похученные при доказательотве те ремн I, получим, что pqд (3) $\left|H, \mu_{n}\right|_{p}$-сукмируек.

Следстви $3[4]$. Еоли $\int_{0}^{\pi}\left|\left(\frac{\psi(t)}{L^{2}}\right)^{\prime}\right|^{p} d t<\infty$, то рад (3) $|E, q|_{1}-$ оуммируем при $t=x$.

Теорема 4. Еоли $\int_{0}^{\pi}\left|\left(\frac{\psi(t)}{t^{2}}\right)^{\prime}\right|^{p} d t<\infty$. . метод $\left(H, \mu_{n}\right)$ хо1. јөрвативен и $\int_{0}^{1} \frac{|d \pi(x)|}{1-x}<\infty$. то ряд (4) $\left|H, \mu_{n}\right|_{p}$-оумкируем прr $t=x$. Дохвзатехьство. LАR ряда (4)

$$
\begin{gathered}
n \ln (x)=\frac{2 n}{\pi} \int_{0}^{\pi} \varphi(t) \cos n t d t= \\
=\frac{4}{\pi^{2}} \frac{\varphi(\pi)}{n} \cos n \pi-\frac{2}{\pi} \int_{0}^{\pi}\left(t^{2} \sin n t+\frac{2 t}{n} \cos n t-\frac{2}{n^{2}} \sin n t\right) d\left(\frac{\varphi(t)}{t^{2}}\right) .
\end{gathered}
$$

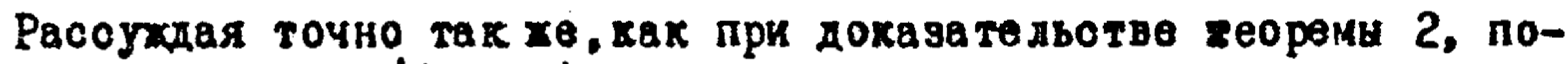
лучим, что рад (4) $\left|H_{1} \mu_{n}\right|_{p}$-оумикруек.

Следствие 4 [4]. Eохи $\int_{0}\left|\left(\frac{\varphi(t)}{t^{2}}\right)^{\prime}\right| d t<\infty$. то pas (4) $|E, q|_{p}-$ сучмиируем при $t=x$. 
Buблиографические ссилки

I. X а р д и Г. Расходяциеся ряды. Mo,I95I.

2. Srivastava P. On the concept of strong summability IIProc. National Inst. Sci. India (A). 1960. Vd. 26.

3. Knopp M. and Lorntz G.G. Baträge zur absoluten Limitie- : rung $/ 1$ archiv der Math. 1949. Vok.2.

4. В : К о а Н.И. Абсолютня степени $P$ суммируенос. р рдов Фурье метопом Эилепа / Иоследования по современнн проблеман оумиировения и ппиолижения функцй и их приложения. Днепропетровеі. 1986.

YIK 5I7. $5 \mathrm{I}$

\section{H.L Човикова}

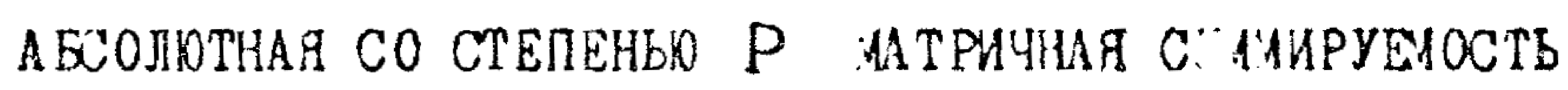

ПOCЛЕД ObATEJ LHOCTEA $\left\{n B_{n}(t)\right\}$

Преобразуеи числовои пяд $\sum U_{k}$ о помон треугоньнои матрицн $T=\left\|a_{n k}\right\|$ следугшим образом:

$$
t_{n}=\sum_{k=0}^{n} a_{n k} S_{k}, \quad S_{n}=\sum_{k=0}^{n} u_{k} .
$$

Говорят, что ряд $\sum$ iк абсолотно со отепеньо $p 1$ оуммируем $\mathrm{T}$-методом или $|\mathrm{T}| \mathrm{p}$-суммируем, если

$$
\sum_{n=0}^{\infty} n^{p-1}\left|t_{n}-t_{n-1}\right|^{p}<\infty \text {. }
$$

Если

$P=\sum_{n=1}^{n} p_{k}=0, \quad k>n$,

$$
a_{n k}=\left\{\begin{array}{cc}
\frac{P_{n-k}}{P_{n}}, & r \leq k \leq n, \\
0, & k>n,
\end{array}\right.
$$

где $\left.P_{n}=\sum_{k=0}^{n} P_{k}=0 . n_{n}\right\}$ - неко трая числовая последователиность, -o похучим метод Bopororo 4ерлунда, еоли $P_{n}=(n+\delta), \delta>0$, nол"чим метод Чеsapo.

Пугть $f(t)-2 \pi$ - периолическая $L$-интегрunyexas нa $(-\pi, \pi)$ 